\title{
Study on Theoretical Model and Test Method of Vertical Vibration of Elevator Traction System
}

\author{
Qifeng Peng, ${ }^{1}$ Aihua Jiang, ${ }^{2}$ Hong Yuan $\mathbb{D D}^{1},{ }^{1}$ Guojian Huang, ${ }^{2}$ Shan He, ${ }^{2}$ and Shanqing Li ${ }^{1}$ \\ ${ }^{1}$ MOE Key Laboratory of Disaster Forecast and Control in Engineering, School of Mechanics and Construction Engineering, \\ Jinan University, Guangzhou 51063, China \\ ${ }^{2}$ Guangzhou Academy of Special Equipment Inspection \& Testing, Guangzhou 510180, China
}

Correspondence should be addressed to Hong Yuan; tyuanhong@jnu.edu.cn and Shanqing Li; lishanqing09@163.com

Received 1 January 2020; Accepted 13 February 2020; Published 14 March 2020

Academic Editor: En-Qiang Lin

Copyright (C) 2020 Qifeng Peng et al. This is an open access article distributed under the Creative Commons Attribution License, which permits unrestricted use, distribution, and reproduction in any medium, provided the original work is properly cited.

In order to study the dynamic characteristics of the elevator, the response characteristics of the elevator under normal operation and emergency braking conditions are analyzed. In this paper, the centralized mass discretization model is used to study the vibration characteristics of the elevator traction system under the external excitation. Firstly, the vibration equation of the elevator multi-degrees-of-freedom (abbreviated as DOF) system is established. Then, the vibration characteristics of the three DOF system are analyzed, and the natural frequencies and modes are obtained. The free vibration equation and forced vibration equation are obtained, and the theoretical solutions are obtained. Finally, the test method proposed in this paper is used to test the normal operation and emergency braking of the elevator. The test results show that, under normal operation conditions, the measured speed and distance errors are not more than $4.2 \%$, the up running distance measured by the elevator is larger than the actual value, while the down running distance is smaller than the actual value; under emergency braking condition, because the steel wire rope can only bear the pulling force, the peak acceleration fluctuation in the up emergency braking process is large, while the down emergency braking acceleration fluctuation is small. Therefore, the elevator vibration analysis model and the vibration test method proposed in this paper can be used for the analysis of elevator dynamic performance, which has great reference value for the safety performance research of the elevator traction system.

\section{Introduction}

As a mean of vertical transportation in buildings, elevators bring great convenience to people's life [1]. With the development of modernization, people's life rhythm is faster and faster, the use range of elevator is gradually expanded, and the use frequency is also higher and higher [2]. Subsequently, the requirements of elevator safety, comfort, and running speed are constantly improved $[3,4]$. How to balance the elevator performance among these three requirements is a problem that elevator workers all over the world have been thinking about. The mechanical analysis of elevator vibration is one of the important means to study this problem. A large number of outstanding scholars have carried out in-depth research on the dynamic characteristics of elevators.

In the process of elevator vibration analysis, according to the different simplified models of the steel wire rope, it is mainly divided into the centralized mass discretization model and the distributed mass continuum model [5].

The centralized mass discretization model is mainly equivalent to the mass-spring-damping system with variable parameters [6]. Watanabe and Okawa studied the vertical vibration of the multi-degree of freedom elevator traction system with the compensation rope. It was considered that the maximum vertical vibration of the system occurred when the elevator broke and analyzed the effect of the brake torque on the vertical displacement of the compensation wheel [7]. Roberts refined the discrete model of the elevator lifting system with centralized parameters, established the 27 DOF model of the elevator lifting system, and verified the consistency between the model and the actual system. Based on the model, the concept of development and evaluation of the high-rise high-speed elevator operation control system 
was proposed [8]. Yang et al. established a four degree of freedom model of elevator lateral vibration, studied the impact of air flow on the system vibration when the ultrahigh speed elevator was running in the shaft, and analyzed the impact of air disturbance on vehicle lateral vibration acceleration under different working conditions by Newmark-beta method [9].

The distributed mass continuum model is that the steel wire rope is equivalent to the extensible beam structure, and the variable length flexible structure analysis theory is used to study. Gaiko and van Horssen regarded the elevator wire rope as a beam with variable length. Assuming the axial vertical velocity of the beam was constant, the lateral vibration of the wire rope under the action of wind vibration was analyzed by singular perturbation theory. The numerical method was also used to verify the calculation results [10]. Fan and Zhu used the singularity free beam to model the elevator steel wire rope, analyzed the natural frequency and vibration mode of the steel wire rope after the change of the elevator car position, and compared with the results of ABAQUS analysis in parallel [11].

In the study of dynamic characteristics of elevator emergency braking process, Durak and Yurtseven studied the tribological properties of an elevator's brake linings and provided information about how to evaluate the tribological properties of the brake linings of the elevator system that were activated in emergencies [12]. Xiao studied the function and importance of elevator brakes and analyzed the causes of brake-related part failure [13]. Poul analyzed the influence of load on effective braking distance and descending speed during emergency braking and gave the calculation results [14]. Peng et al. studied a test method of no-load elevator braking torque and gave a method to predict full-load braking capacity based on noload test results [15]. Longwic and Szydło introduced the test results of the influence of guide rail pollution on the braking delay of the progressive gear in the friction lift. The influence of lubrication on braking performance was studied [16].

These research works provide an important reference for the vibration research of the elevator traction system, but most scholars focus on the lateral vibration analysis of the steel wire rope and the lift car. In the process of simplification, the contact between the lift car side (or the counterweight side) wire rope and the traction wheel is simplified as a fixed connection, but less attention is paid to the influence of the rotation of the traction wheel and the slip characteristics of the wire rope. In this paper, based on the actual operation characteristics of the elevator, the vibration of the elevator traction system is studied by using the centralized parameter discrete model, and the influence of the rotation characteristics of the traction wheel is considered. The dynamic characteristics of the elevator traction system in the normal operation and emergency braking process are studied by using the theoretical solution and experimental methods for comparison.

\section{Research on the Centralized Mass Discretization Model of the Elevator Traction System}

When the elevator is powered off or the safety device acts, the elevator brake will hold the brake and stop the elevator to ensure the safety of the passengers [17-19]. In the process of emergency braking, due to the effect of the brake shoe, the whole elevator traction system will have strong vibration.

In order to analyze the influence of braking moment on the lift car and counterweight, a 1:1 elevator model is studied in this paper, the centralized mass discretization model is used to analyze the vibration response of the elevator traction system under the action of external torque, and the influence of the relative slip between the wire rope and the traction wheel is also considered.

The elevator traction system discussed in this paper (as shown in Figure 1) includes traction the wheel, traction wire rope, guide wheel, reverse rope wheel, lift car, counterweight, compensation rope, and tension wheel. During the analysis of vibration characteristics, the following assumptions are made.

\subsection{Basic Assumptions}

(1) It is assumed that each wire rope of the elevator is always in a state of tension with uniform force

(2) It is assumed that multiple steel wire ropes can be regarded as one rope, and the cross-sectional area of this rope is equal to the sum of the cross-sectional areas of the wire rope

(3) It is assumed that the deformation of the steel wire rope conforms to Hooke's law, and its elastic modulus remains unchanged along the whole length of the wire rope

(4) The elasticity between the foundation and the tractor is not considered

(5) The lateral vibration of the traction system caused by the guide rail and other ancillary facilities is not considered

(6) The cage and counterweight are regarded as rigid bodies, and the traction wheel and tension wheel are regarded as rigid pulleys

(7) Ignoring the elasticity and quality of the wire rope in close contact with the traction wheel and tension wheel.

2.2. Energy Description of the Elevator Traction System. The steel wire rope is divided into 4 sections (as shown in Figure 2), including cage side traction rope $L_{1}$, counterweight side traction rope $L_{2}$, cage side compensation rope $L_{3}$, counterweight side compensation rope $L_{4}$, wire rope on traction wheel $L_{5}$, and wire rope on tension wheel $L_{6}$. $L_{1}$ is divided into $n_{1}$ segments, the stiffness of the spring in the $i$ th segment is $k_{1, i}$, the damping is $c_{1, i}$, the mass is simplified to $n_{1}+1$ particles, and the mass of the $i$ th particle is $m_{1, i}$. $L_{2}$ is 


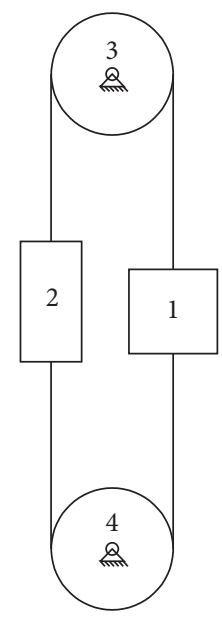

FIGURE 1: Simplified drawing of the elevator traction system. (1) Lift car, (2) counterweight, (3) traction wheel, and (4) tension wheel.

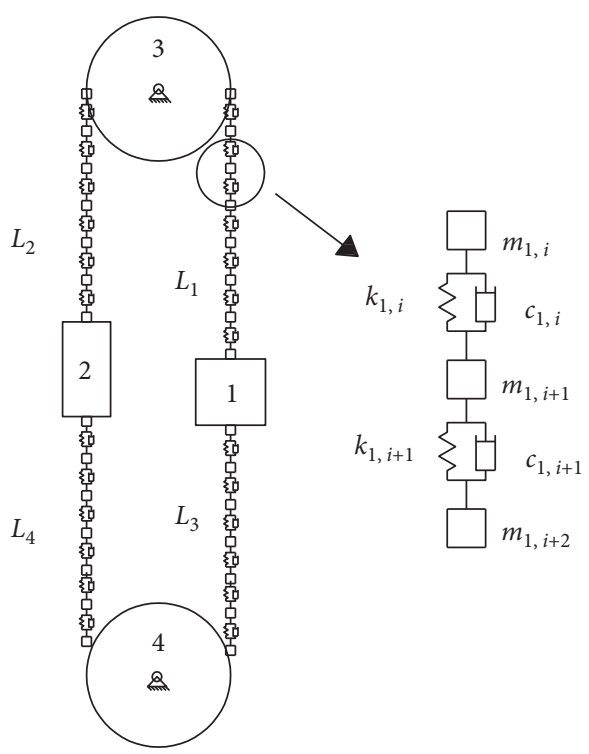

Figure 2: Mass-spring-damping diagram of the elevator traction system.

divided into $n_{2}$ segments, the stiffness of the spring in the $i$ th segment is $k_{2, i}$, the damping is $c_{2, i}$, the mass is simplified to $n_{2}+1$ particles, and the mass of the $i$ th particle is $m_{2, i}$. $L_{3}$ is divided into $n_{3}$ segments, the stiffness of the spring in the $i$ th segment is $k_{3, i}$, the damping is $c_{3, i}$, the mass is simplified to $n_{3}+1$ particles, and the mass of the $i$ th particle is $m_{3, i} . L_{4}$ is divided into $n_{4}$ segments, the stiffness of the spring in the $i$ th segment is $k_{4, i}$, the damping is $c_{4, i}$, the mass is simplified to $n_{4}+1$ particles, and the mass of the $i$ th particle is $m_{4, i}$.

The kinetic energy of the $i$ th particle of $L_{1}$ is

$$
T_{1, i}=\frac{1}{2} m_{1, i} \dot{x}_{1, i}^{2}
$$

The total kinetic energy of $L_{1}$ is

$$
T_{1}=\frac{1}{2} \sum_{i=0}^{n_{1}} m_{1, i} \dot{x} \cdot{ }^{2} .
$$

Similarly, it can be known that the total kinetic energy of $L_{2}$ is

$$
T_{2}=\frac{1}{2} \sum_{i=0}^{n_{2}} m_{2, i} \dot{x}_{2, i}^{2}
$$

The total kinetic energy of $L_{3}$ is

$$
T_{3}=\frac{1}{2} \sum_{i=0}^{n_{3}} m_{3, i} \dot{x_{3, i}}{ }^{2}
$$

The total kinetic energy of $L_{4}$ is

$$
T_{4}=\frac{1}{2} \sum_{i=0}^{n_{4}} m_{4, i} \dot{x}_{4, i}^{2}
$$

The cage displacement is represented by the generalized coordinate $x_{c}$, the counterweight displacement is represented by the generalized coordinate $x_{w}$, the rotation angle of the traction wheel is represented by $x_{T r}$, and the rotation angle of the tension wheel is represented by $x_{\mathrm{Te}}$. Because of the coincidence of marks, there are $x_{1, n_{1}}=x_{c}=x_{3,0}$, $x_{2, n_{2}}=x_{w}=x_{4,0}, \quad R_{\mathrm{Tr}} x_{\mathrm{Tr}}=x_{2,0}+x_{1,0}, \quad$ and $\quad R_{\mathrm{Te}} x_{\mathrm{Te}}=$ $-x_{3, n_{3}}-x_{4, n_{4}}$.

The sum of kinetic energy of the cage, counterweight, traction wheel, and tension wheel is

$$
\begin{aligned}
T_{5}= & \frac{1}{2} m_{c} \dot{x}_{c}^{2}+\frac{1}{2} m_{w} \cdot{ }^{2}+\frac{1}{2} J_{\mathrm{Tr}}{\dot{x_{\mathrm{Tr}}}}^{2}+\frac{1}{2} J_{\mathrm{Te}}{\dot{x_{\mathrm{Te}}}}^{2}=\frac{1}{2} m_{c} \dot{x_{1, n_{1}}}{ }^{2} \\
& +\frac{1}{2} m_{w} \dot{x_{2, n_{2}}}{ }^{2}+\frac{1}{2 R_{\mathrm{Tr}}{ }^{2}} J_{\mathrm{Tr}}\left(\dot{x_{2,0}}+\dot{x_{1,0}}\right)^{2}+\frac{1}{2 R_{\mathrm{Te}}{ }^{2}} J_{\mathrm{Te}}\left(\dot{x_{3, n_{3}}}+\dot{x}_{4, n_{4}}\right)^{2} .
\end{aligned}
$$

The total kinetic energy of the traction system is

$$
\begin{aligned}
T= & \frac{1}{2} \sum_{i=0}^{n_{1}} m_{1, i} \dot{x}_{1, i}{ }^{2}+\frac{1}{2} \sum_{i=0}^{n_{2}} m_{2, i} \dot{x_{2, i}}{ }^{2}+\frac{1}{2} \sum_{i=0}^{n_{3}} m_{3, i} \dot{x_{3, i}}{ }^{2}+\frac{1}{2} \sum_{i=0}^{n_{4}} m_{4, i} \dot{x_{4, i}}{ }^{2} \\
& +\frac{1}{2} m_{c} \dot{x_{1, n_{1}}}{ }^{2}+\frac{1}{2} m_{w} \dot{x_{2, n_{2}}}{ }^{2}+\frac{1}{2 R_{\mathrm{Tr}}^{2}} J_{\mathrm{Tr}}\left(\dot{x_{2,0}}+\dot{x}_{1,0}\right)^{2} \\
& +\frac{1}{2 R_{\mathrm{Te}}^{2}} J_{\mathrm{Te}}\left(\dot{x_{3, n_{3}}}+\dot{x_{4, n_{4}}}\right)^{2} .
\end{aligned}
$$

As a vertical suspension system, the movement of the elevator in the hoistway is affected by the damping force. To accurately analyze the dynamic characteristics of the elevator, the damping force is a factor that cannot be ignored.

The energy dissipation function of the wire rope in section I of $L_{1}$ can be expressed as

$$
D_{1, i}=\frac{1}{2} c_{1, i}\left(\dot{x_{1, i}}-x_{1, i-1}\right)^{2} .
$$

The total energy dissipation function of $L_{1}$ can be expressed as

$$
D_{1}=\frac{1}{2} \sum_{i=1}^{n_{1}} c_{1, i}\left(\dot{x_{1, i}}-x_{1, i-1}\right)^{2} .
$$

Similarly, the total energy dissipation function of $L_{2}$ can be expressed as 


$$
D_{2}=\frac{1}{2} \sum_{i=1}^{n_{2}} c_{2, i}\left(\dot{x_{2, i}}-x_{2, i-1}\right)^{2}
$$

The total energy dissipation function of $L_{3}$ can be expressed as

$$
D_{3}=\frac{1}{2} \sum_{i=1}^{n_{3}} c_{3, i}\left(\dot{x_{3, i}}-x_{3, i-1}\right)^{2}
$$

The total energy dissipation function of $L_{4}$ can be expressed as

$$
D_{4}=\frac{1}{2} \sum_{i=1}^{n_{4}} c_{4, i}\left(\dot{x_{4, i}}-x_{4, i-1}\right)^{2}
$$

Since $x_{1, n_{1}}=x_{c}=x_{3,0}$ and $x_{2, n_{2}}=x_{w}=x_{4,0}$, the work done by the damping force of the car and counterweight is reflected in the energy dissipation function of the wire rope. The energy dissipation function of the traction wheel and the tension wheel rotation can be expressed as

$$
\begin{aligned}
D_{5}= & \frac{1}{2} c_{\mathrm{Tr}} x_{\mathrm{Tr}}^{2}+\frac{1}{2} c_{\mathrm{Te}} x_{\mathrm{Te}}^{2}=\frac{1}{2 R_{\mathrm{Tr}}^{2}} c_{\mathrm{Tr}}\left(x_{2,0}^{\cdot}+\dot{x}_{1,0}^{\cdot}\right)^{2} \\
& +\frac{1}{2 R_{\mathrm{Te}}^{2}} c_{\mathrm{Te}}\left(x_{3, n_{3}}+x_{4, n_{4}}\right)^{2} .
\end{aligned}
$$

The total energy dissipation function of the elevator traction system is

$$
\begin{aligned}
D= & \frac{1}{2} \sum_{i=1}^{n_{1}} c_{1, i}\left(\dot{x_{1, i}}-x_{1, i-1}\right)^{2}+\frac{1}{2} \sum_{i=1}^{n_{2}} c_{2, i}\left(\dot{x_{2, i}}-x_{2, i-1}\right)^{2} \\
& +\frac{1}{2} \sum_{i=1}^{n_{3}} c_{3, i}\left(\dot{x_{3, i}}-x_{3, i-1}\right)^{2}+\frac{1}{2} \sum_{i=1}^{n_{4}} c_{4, i}\left(\dot{x_{4, i}}-x_{4, i-1}\right)^{2} \\
& +\frac{1}{2 R_{\mathrm{Tr}}^{2}} c_{\mathrm{Tr}}\left(\dot{x}_{2,0}+\dot{x}_{1,0}\right)^{2}+\frac{1}{2 R_{\mathrm{Te}}^{2}} c_{\mathrm{Te}}\left(x_{3, n_{3}}+x_{4, n_{4}}\right)^{2}
\end{aligned}
$$

The potential energy of the elevator traction system can be expressed as

$$
\begin{aligned}
U= & \frac{1}{2} \sum_{i=1}^{n_{1}} k_{1, i}\left(x_{1, i}-x_{1, i-1}\right)^{2}+\frac{1}{2} \sum_{i=1}^{n_{2}} k_{2, i}\left(x_{2, i}-x_{2, i-1}\right)^{2} \\
& +\frac{1}{2} \sum_{i=1}^{n_{3}} k_{3, i}\left(x_{3, i}-x_{3, i-1}\right)^{2}+\frac{1}{2} \sum_{i=1}^{n_{4}} k_{4, i}\left(x_{4, i}-x_{4, i-1}\right)^{2} .
\end{aligned}
$$

The total number of degrees of freedom (abbreviated as $\mathrm{DOF})$ of the system is $n_{1}+n_{2}+n_{3}+n_{4}$. According to Lagrange theory, the motion equation of the vertical vibration system of the elevator system can be expressed as

$$
\frac{\mathrm{d}}{\mathrm{d} t}\left(\frac{\partial T}{\partial \dot{x_{j, i}}}\right)-\frac{\partial T}{\partial x_{j, i}}+\frac{\partial U}{\partial x_{j, i}}+\frac{\partial D}{\partial \dot{x}_{j, i}}=Q_{i}, \quad j=1,2,3,4
$$

$$
i=1,2,3, \ldots, n_{i} \text {. }
$$

\section{Establishment and Solution of the Vertical Vibration Equation of the 3-DOF System}

There is no compensation rope (or compensation chain) for elevators with low lift height. Compared with the cage mass, the quality of the elevator wire rope can be equivalent to the cage by Rayleigh method [20]. In this way, the elevator traction and the hoisting system can be simplified into a three-degree-of-freedom system composed of the cage, the weight, and the traction wheel.

3.1. Energy Description of the 3-DOF System. The lift car displacement is represented by $x_{1}$, counterweight displacement is represented by $x_{2}$, and the rotation angle of the traction wheel is represented by $x_{3}$, as shown in Figure 3.

$$
\begin{aligned}
& T=\frac{1}{2} m_{c} \dot{x_{1}^{2}}+\frac{1}{2} m_{w} \dot{x_{2}^{2}}+\frac{1}{2} J_{\mathrm{Tr}} \dot{x}_{3}^{2}, \\
& D=\frac{1}{2} C_{c}\left(\dot{x_{1}}+R_{\mathrm{Tr}} \dot{x_{3}}\right)^{2}+\frac{1}{2} C_{w}\left(\dot{x_{2}}-R_{\mathrm{Tr}} \dot{x_{3}}\right)^{2}, \\
& U=\frac{1}{2} k_{c}\left(x_{1}+R_{\mathrm{Tr}} x_{3}\right)^{2}+\frac{1}{2} k_{w}\left(x_{2}-R_{\mathrm{Tr}} x_{3}\right)^{2} .
\end{aligned}
$$

For the generalized coordinate $x_{1}$, we have

$$
\begin{aligned}
\frac{\mathrm{d}}{\mathrm{d} t}\left(\frac{\partial T}{\partial \dot{x_{1}}}\right) & =m_{c} \ddot{x}_{2}, \\
\frac{\partial T}{\partial x_{1}} & =0, \\
\frac{\partial U}{\partial x_{1}} & =k_{c}\left(x_{1}+R_{\mathrm{Tr}} x_{3}\right), \\
\frac{\partial D}{\partial \dot{x_{1}}} & =C_{c}\left(\dot{x}_{1}+R_{\mathrm{Tr}} \dot{x_{3}}\right) .
\end{aligned}
$$

The elevator cage is only affected by wire rope tension and gravity in the vertical direction, and by substituting the above results into Lagrange equation, we can get

$$
m_{c} \ddot{x}_{1}+C_{c}\left(\dot{x_{1}}+R_{\mathrm{Tr}} \dot{x}_{3}\right)+k_{c}\left(x_{1}+R_{\mathrm{Tr}} x_{3}\right)=0 .
$$

For the generalized coordinate $x_{2}$, we have

$$
\begin{aligned}
\frac{\mathrm{d}}{\mathrm{d} t}\left(\frac{\partial T}{\partial \dot{x_{2}}}\right) & =m_{w} \ddot{x}_{2}, \\
\frac{\partial T}{\partial x_{2}} & =0, \\
\frac{\partial U}{\partial x_{2}} & =k_{w}\left(x_{2}-R_{\operatorname{Tr}} x_{3}\right), \\
\frac{\partial D}{\partial \dot{x_{2}}} & =C_{w}\left(\dot{x_{2}}-R_{\operatorname{Tr}} \dot{x_{3}}\right) .
\end{aligned}
$$

The elevator counterweight is only affected by wire rope tension and gravity in the vertical direction, and by substituting the above results into Lagrange equation, we can get

$$
m_{w} \ddot{x}_{2}+C_{w}\left(\dot{x}_{2}-R_{\mathrm{Tr}} \dot{x}_{3}\right)+k_{w}\left(x_{2}-R_{\mathrm{Tr}} x_{3}\right)=0 .
$$




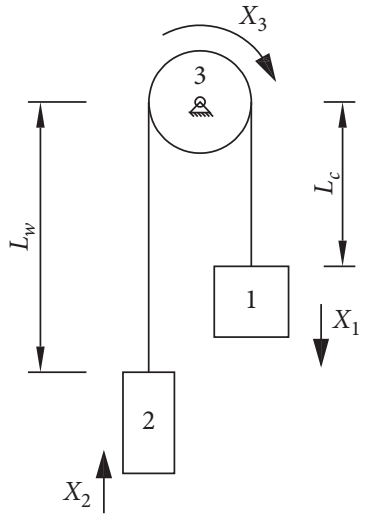

FIGURE 3: Mass-spring-damping diagram of the three-degree-offreedom system.

For the generalized coordinate $x_{3}$, we have

$$
\begin{aligned}
\frac{\mathrm{d}}{\mathrm{d} t}\left(\frac{\partial T}{\partial \dot{x_{3}}}\right) & =J_{\mathrm{Tr}} \ddot{x}_{3}, \frac{\partial T}{\partial x_{3}}=0, \\
\frac{\partial U}{\partial x_{3}} & =k_{c} R_{\mathrm{Tr}}\left(x_{1}+R_{\mathrm{Tr}} x_{3}\right)-k_{w} R_{\mathrm{Tr}}\left(x_{2}-R_{\mathrm{Tr}} x_{3}\right), \\
\frac{\partial D}{\partial \dot{x}_{3}} & =C_{c} R_{\mathrm{Tr}}\left(\dot{x}_{1}+R_{\mathrm{Tr}} \dot{x}_{3}\right)-C_{w} R_{\mathrm{Tr}}\left(\dot{x}_{2}-R_{\mathrm{Tr}} \dot{x}_{3}\right) .
\end{aligned}
$$

The external torque $M(T)$ is applied to the traction wheel, so

$$
\begin{gathered}
J_{\mathrm{Tr}} \ddot{x}_{3}+C_{c} R_{\mathrm{Tr}} \dot{x}_{1}-C_{w} R_{\mathrm{Tr}} \dot{x}_{2}+\left(C_{c} R_{\mathrm{Tr}}^{2}+C_{w} R_{\mathrm{Tr}}{ }^{2}\right) \dot{x}_{3}+k_{c} R_{\mathrm{Tr}} x_{1} \\
-k_{w} R_{\mathrm{Tr}} x_{2}+\left(k_{c} R_{\mathrm{Tr}}^{2}+k_{w} R_{\mathrm{Tr}}^{2}\right) x_{3}=M(t) .
\end{gathered}
$$

3.2. Establishment of Differential Equations of Motion. Equations (19), (21), and (23) constitute the differential equation of motion of the traction system, which can be expressed in the matrix form as follows:

$$
[M]\{\ddot{x}\}+[C]\{\dot{x}\}+[K]\{x\}=\{F\},
$$

where

$$
\begin{aligned}
& \{x\}=\left\{\begin{array}{lll}
x_{1} & x_{2} & x_{3}
\end{array}\right\}^{T}, \\
& {[M]=\left[\begin{array}{ccc}
m_{c} & 0 & 0 \\
0 & m_{w} & 0 \\
0 & 0 & J_{\mathrm{Tr}}
\end{array}\right] \text {, }} \\
& {[C]=\left[\begin{array}{ccc}
C_{c} & 0 & C_{c} R_{\mathrm{Tr}} \\
0 & C_{w} & -C_{w} R_{\mathrm{Tr}} \\
C_{c} R_{\mathrm{Tr}} & -C_{w} R_{\mathrm{Tr}} & C_{c} R_{\mathrm{Tr}}^{2}+C_{w} R_{\mathrm{Tr}}^{2}
\end{array}\right] \text {, }} \\
& {[K]=\left[\begin{array}{ccc}
k_{c} & 0 & k_{c} R_{\mathrm{Tr}} \\
0 & k_{w} & -k_{w} R_{\mathrm{Tr}} \\
k_{c} R_{\mathrm{Tr}} & -k_{w} R_{\mathrm{Tr}} & k_{c} R_{\mathrm{Tr}}^{2}+k_{w} R_{\mathrm{Tr}}^{2}
\end{array}\right] \text {, }} \\
& {[F]=\left\{\begin{array}{lll}
0 & 0 & M(t)
\end{array}\right\}^{T} \text {. }}
\end{aligned}
$$

3.3. Free Vibration Solution of the Undamped System. Solving equation (24), for the free vibration of the undamped system,

$$
[M]\{\ddot{x}\}+[K]\{x\}=\{0\} .
$$

The characteristic equation of the undamped system is

$$
\left|[K]-\omega_{n}^{2}[M]\right|=0 .
$$

The characteristic roots $\omega_{n 1}, \omega_{n 2}$, and $\omega_{n 3}$ of the system are obtained, where $\omega_{n 1}<\omega_{n 2}<\omega_{n 3}$.

Substituting $\omega_{n 1}$ into the characteristic problem equation of the system, we have

$$
\left([K]-\omega_{n}^{2}[M]\right)[u]=[0] .
$$

Then,

$$
u_{1}^{(1)}=\left\{\begin{array}{c}
u_{1}^{(1)} \\
u_{2}^{(1)} \\
u_{3}^{(1)}
\end{array}\right\}
$$

Similarly, the second and third modal vectors of the undamped system are $\left\{u^{(2)}\right\}$ and $\left\{u^{(3)}\right\}$, respectively.

The modal matrix of the system is obtained as

$$
[u]=\left[\left\{u^{(1)}\right\},\left\{u^{(2)}\right\},\left\{u^{(3)}\right\}\right] .
$$

The modal mass matrix of the system is

$$
\left[M_{r}\right]=[u]^{T}[M][u]=\left[\begin{array}{lll}
M_{r 1} & & \\
& M_{r 2} & \\
& & M_{r 3}
\end{array}\right] .
$$

The modal stiffness matrix of the system is

$$
\left[K_{r}\right]=[u]^{T}[K][u]=\left[\begin{array}{lll}
K_{r 1} & & \\
& K_{r 2} & \\
& & K_{r 3}
\end{array}\right] .
$$

The ratio of the modal mass $M_{\text {ri }}$ to the modal stiffness $K_{\text {ri }}$ is equal to the square of the natural frequency $\omega_{\text {ni }}$ of the same order:

$$
\omega_{\mathrm{ni}}^{2}=\frac{K_{\mathrm{ri}}}{M_{\mathrm{ri}}}
$$

Since the damping of the elevator traction system is small, Rayleigh damping can be used to deal with the system damping, that is, the damping is assumed to be a linear combination of mass and stiffness:

$$
[C]=\alpha[M]+\beta[K] .
$$

Then, the damping ratio of the $i$ th mode is

$$
\zeta_{i}=\frac{\alpha+\beta \omega_{\mathrm{ni}}^{2}}{2 \omega_{\mathrm{ni}}} .
$$

The modal damping matrix is 


$$
\left[C_{r}\right]=[u]^{T}[C][u]=\left[\begin{array}{lll}
2 \zeta_{1} \omega_{n 1} M_{r 1} & & \\
& 2 \zeta_{2} \omega_{n 2} M_{r 2} & \\
& 2 \zeta_{3} \omega_{n 3} M_{r 3}
\end{array}\right] .
$$

3.4. Regularization. In order to carry out the analysis and calculation more clearly, the decoupled differential equation of system motion is regularized, and the regularization factor of order $i$ is carried out:

$$
\alpha_{\mathrm{ri}}=\frac{1}{\sqrt{M_{\mathrm{ri}}}} .
$$

We get the regularized factor matrix

$$
\left[\alpha_{r}\right]=\left[\begin{array}{lll}
\alpha_{r 1} & & \\
& \alpha_{r 2} & \\
& & \alpha_{r 3}
\end{array}\right] .
$$

The regularized mode vector of order $i$ is

$$
\left\{u_{N}^{(i)}\right\}=\alpha_{\text {ri }}\left\{u^{(i)}\right\} .
$$

The regularized mode matrix is

$$
\left[u_{N}\right]=\left[\left\{u_{N}^{(1)}\right\},\left\{u_{N}^{(2)}\right\},\left\{u_{N}^{(3)}\right\}\right]=[u]\left[\alpha_{r}\right] .
$$

Regularized mode mass is

$$
M_{\mathrm{Ni}}=\left\{u_{N}^{(i)}\right\}^{T}[M]\left\{u_{N}^{(i)}\right\}=1 .
$$

Regularized modal stiffness is

$$
K_{\mathrm{Ni}}=\left\{u_{N}^{(i)}\right\}^{T}[K]\left\{u_{N}^{(i)}\right\}=\omega_{\mathrm{ni}}^{2} .
$$

The regular mode matrix is used to transform the physical coordinates of the system:

$$
\{x(t)\}=\left[u_{N}\right]\left\{q_{N}(t)\right\} .
$$

The system vibration equation is transformed into a regularized mode equation:

$$
\left.\left\{q_{N} \ddot{(} t\right)\right\}+\left[2 \zeta_{i} \omega_{\text {ni }}\right]\left\{\dot{q_{N}}(t)\right\}+\left[\omega_{\text {ni }}^{2}\right]\left\{q_{N}(t)\right\}=\{N(t)\},
$$

where $q_{N}(t)$ is the regularized modal coordinates, and the elements in the damping matrix and the stiffness matrix represent any element on the diagonal; $\{N(t)\}$ represents the generalized excitation force vector after regularization as follows:

$$
\{N(t)\}=\left[u_{N}\right]^{T}\{F(t)\}=\left\{N_{1}(t), N_{2}(t), N_{3}(t)\right\}^{T},
$$

where

$$
\begin{aligned}
N_{i}(t) & =\left\{u_{N}^{(i)}\right\}^{T}\{F(t)\}=F_{1}(t) u_{N 1}^{(i)}+F_{2}(t) u_{N 2}^{(i)}+F_{3}(t) u_{N 3}^{(i)} \\
& =\sum_{j=1}^{3} F_{j}(t) u_{\mathrm{Nj}}^{(i)} .
\end{aligned}
$$

Then, the regularized modal equation can be expanded to

$$
q_{\mathrm{Ni}} \ddot{(}(t)+2 \zeta_{i} \omega_{\mathrm{ni}} q_{\mathrm{Ni}}^{\dot{2}}(t)+\omega_{\mathrm{ni}}^{2} q_{\mathrm{Ni}}(t)=N_{i}(t), \quad i=1,2,3 .
$$

3.5. Solution of the Regular Modal Equation. For the elevator traction system, the external force on the system is

$$
\begin{aligned}
\{F(t)\} & =\left\{\begin{array}{lll}
0 & 0 & M(t)
\end{array}\right\}, \\
M(t) & = \begin{cases}M_{m}, & t \leq t_{1}, \\
M_{s}, & t>t_{1},\end{cases}
\end{aligned}
$$

where $M_{m}$ is the value of dynamic friction torque, $M_{s}$ is the value of static friction torque, and both values are assumed to be a constant.

When the side mass of the elevator cage is less than the opposite mass, the emergency braking situation of the elevator cage descending is analyzed.

$$
\begin{aligned}
M_{m} & =m_{c}(a+g) R_{\mathrm{Tr}}-m_{w}(g-a) R_{\mathrm{Tr}}, \\
M_{s} & =m_{c} g R_{\mathrm{Tr}}-m_{w} g R_{\mathrm{Tr}} .
\end{aligned}
$$

Equation (45) can be written as follows:

$$
\{N(t)\}=\left[u_{N}\right]^{T}\{F(t)\}=\left\{N_{1}(t), N_{2}(t), N_{3}(t)\right\}^{T} .
$$

The expansion of the regular mode equation is

$$
\ddot{q}_{\mathrm{Ni}}(t)+2 \zeta_{i} \omega_{\mathrm{ni}} \dot{q}_{\mathrm{Ni}}(t)+\omega_{\mathrm{ni}}^{2} q_{\mathrm{Ni}}(t)=N_{i}, \quad i=1,2,3 .
$$

For $\omega_{n 1}=0$, there is

$$
q_{N 11}=0 .
$$

For $\omega_{\mathrm{ni}} \neq 0$, using Duhamel's integral, the response of the system to arbitrary excitation $N_{i}(t)$ can be known as

$$
\begin{aligned}
q_{\mathrm{Ni1}}(t)= & \int_{0}^{t} N_{i}(\tau) h(t-\tau) \mathrm{d} \tau=\frac{1}{\omega_{\mathrm{di}}} \int_{0}^{t} N_{i}(\tau) e^{-\zeta_{i} \omega_{\mathrm{ni}}(t-\tau)} \\
& \cdot \sin \omega_{\mathrm{di}}(t-\tau) \mathrm{d} \tau,
\end{aligned}
$$

where

$$
\omega_{\mathrm{di}}=\sqrt{1-\zeta_{i}^{2}} \omega_{\mathrm{ni}} .
$$

Solve the free vibration equation of the damped system. The external load is 0 in the case of free vibration.

$$
\{F(t)\}=\{0\} .
$$

Then, equation (45) can be written as follows:

$$
\{N(t)\}=\left[u_{N}\right]^{T}\{F(t)\}=\{0\} .
$$

The expansion of the regular mode equation is

$$
q_{\mathrm{Ni}}(t)+2 \zeta_{i} \omega_{\mathrm{ni}} \dot{q_{\mathrm{Ni}}}(t)+\omega_{\mathrm{ni}}^{2} q_{\mathrm{Ni}}(t)=0, \quad i=1,2,3 .
$$

For $\omega_{n 1}=0$, we have 


$$
q_{N 12}=C_{1} t+D_{1} \text {. }
$$

For $\omega_{\text {ni }} \neq 0$, the solution of the canonical modal equation is obtained by solving the differential equation of free vibration of a single degree of freedom.

$$
q_{\mathrm{Ni} 2}(t)=e^{-\zeta_{i} \omega_{\mathrm{ni}} t}\left(C_{i} \cos \omega_{\mathrm{di}} t+D_{i} \sin \omega_{\mathrm{di}} t\right) .
$$

The general solution of equation (51) is

$$
q_{\mathrm{Ni}}(t)=q_{\mathrm{Ni1}}(t)+q_{\mathrm{Ni} 2}(t) .
$$

3.6. Coordinate Substitution. Substituting $q_{N 1}(t), q_{N 2}(t)$, and $q_{N 3}(t)$ into coordinate transformation equation (43), the corresponding physical coordinate solution of the system is obtained as follows:

$$
\{x(t)\}=\left[u_{N}\right]\left\{q_{N}(t)\right\} .
$$

\section{Study on the Vertical Vibration Method of the Elevator Traction System}

The normal operation conditions of the elevator mainly include normal start, running at a normal uniform speed, normal stop, and emergency braking [21, 22]. Emergency braking condition requires much higher braking performance than normal braking. On the one hand, the elevator brake suddenly holds the brake during the emergency braking, which has a great impact on the tractor and the traction wheel [23]. On the other hand, for the elevator car, the speed reduction caused by emergency braking will have a greater impact on the elevator car, affecting the ride comfort and safety of the elevator [24].

4.1. Model Parameters. For the vertical vibration system of the traction elevator, the external force is applied only at the traction wheel. This force is the driving torque of the tractor under normal operating conditions and the braking torque of the brake $M(t)$ during stop or stationary state. An emergency braking test method of the elevator traction system is studied in this paper, which is used to test the acceleration change of the elevator cage during emergency braking.

The parameters of the elevator are shown in Table 1.

The value range of elastic modulus $E$ of the steel wire rope is $8 \times 10^{8} \mathrm{~N} / \mathrm{m}^{2} \sim 1.5 \times 10^{11} \mathrm{~N} / \mathrm{m}^{2}$ [25]. Considering the elastic characteristics of the elevator steel wire rope, this paper takes $E=8 \times 10^{8} \mathrm{~N} / \mathrm{m}^{2}$.

Elastic coefficient of the cage side wire rope is

$$
k_{c}=\frac{\mathrm{EA}}{L_{c}}=\frac{\pi \mathrm{En}_{R} d_{R}^{2}}{4 L_{c}}
$$

where $L_{c}$ is the length of the wire rope on the side of the cage.

\begin{tabular}{|c|c|c|c|}
\hline Nomenclature & Parameter & Value & Unit \\
\hline$m_{c}$ & Masses of the car & 1070 & $\mathrm{~kg}$ \\
\hline$m_{w}$ & Masses of the counterweight & 1526 & $\mathrm{~kg}$ \\
\hline$J_{\operatorname{Tr}}$ & $\begin{array}{c}\text { Equivalent moment of inertia } \\
\text { of the } \\
\text { traction wheel }\end{array}$ & 12.5 & $\mathrm{~kg} \cdot \mathrm{m}^{2}$ \\
\hline$\zeta_{1}$ & First mode damping ratio & 0.04 & - \\
\hline$\zeta_{2}$ & Second mode damping ratio & 0.06 & - \\
\hline$R_{\mathrm{Tr}}$ & Radius of the traction wheel & 0.225 & $\mathrm{~m}$ \\
\hline E & $\begin{array}{l}\text { Elastic modulus of a single } \\
\text { wire rope }\end{array}$ & $1 \times 10^{11}$ & $\mathrm{~N} / \mathrm{m}^{2}$ \\
\hline$d_{R}$ & Wire rope diameter & $10 \times 10^{-3}$ & $\mathrm{~m}$ \\
\hline$n_{R}$ & Wire rope number & 5 & - \\
\hline $\mathrm{H}$ & Total length of the wire rope & 10 & $\mathrm{~m}$ \\
\hline
\end{tabular}

Elastic coefficient of the counterweight side wire rope is

$$
k_{w}=\frac{\mathrm{EA}}{L_{w}}=\frac{\pi \mathrm{En}_{R} d_{R}^{2}}{4\left(H-L_{c}\right)},
$$

TABLE 1: Model parameter values.

where $L_{w}$ is the length of the wire rope on the side of the counterweight, and $L_{w}=H-L_{c}$.

4.2. Experimental Method. The experimental steps are as follows:

(1) Measure the diameter of the traction wheel, the diameter of the wire rope, and the mass of the cage and counterweight

(2) Arrange the vibration testing instrument on the top of the car [15], the parameters of the test instruments and sensors used are shown in Table 2, and the position and fixing of the acceleration sensor are shown in Figure 4

(3) Professional maintenance staff shall carry out the following operations in the elevator room:

(a) Call the elevator to the bottom floor

(b) Start the elevator normally and go up to the constant speed running stage

(c) When the elevator goes up to the middle of the hoistway, the power will be cut off and the elevator will stop in emergency

(d) Restore the elevator to normal operation, and call the elevator to the top floor

(e) Start the elevator normally and go down to the constant speed running stage

(f) When the elevator goes down to the middle of the hoistway, the power will be cut off and the elevator will stop in emergency

(g) Restore the elevator to normal operation, and let the elevator go back to the ground floor

4.3. Working Condition Division. The measured curve integration of normal operation and emergency braking of the elevator is shown in Figure 5.

The above test results can be divided into four working conditions, which are (1) Condition I: normal downward working condition; (2) Condition II: upward emergency stop condition; (3) Condition III: normal upward working condition; and (4) Condition IV: downward emergency stop 
TABLE 2: ASC acceleration sensor.

\begin{tabular}{lc}
\hline Manufacturer & ASC GmbH \\
\hline Type & ASC 4421-001-6A \\
Country & Germany \\
Serial number & $16-23872$ \\
\hline
\end{tabular}

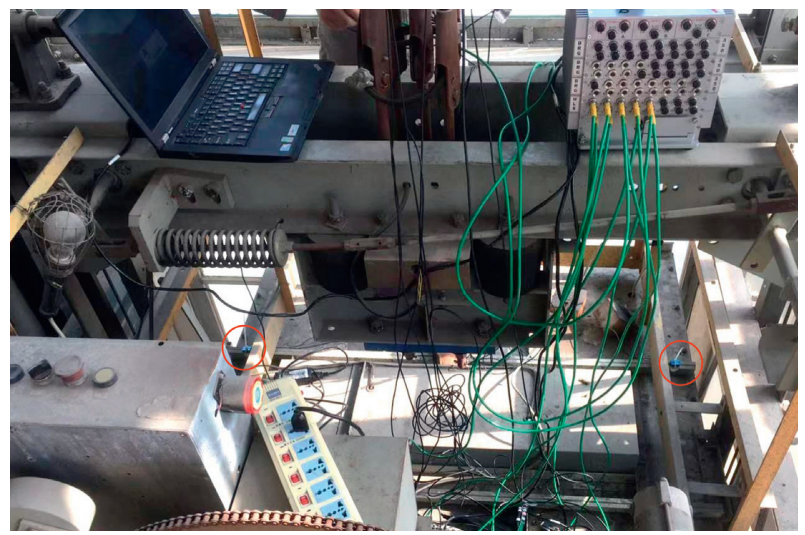

FIgURE 4: Fixing of the acceleration sensor.

condition. The changes of acceleration, velocity, and displacement under these four working conditions are analyzed as follows.

4.4. Test Results of Condition I. Condition I: normal downward working condition. This condition is divided into 3 phases, and they are P.1 acceleration phase, P.2 constant speed phase, and P.3 deceleration phase, Figure 6 .

The normal operation condition referred to in this paper is that the elevator car runs from the top to the bottom (condition I) or from the bottom to the top (condition III) at the rated speed. The rated speed of the elevator is $1 \mathrm{~m} / \mathrm{s}$, and the actual distance from the highest to the lowest floor is $7.513 \mathrm{~m}$ measured by the laser rangefinder. The measured acceleration can be integrated once to obtain the running speed curve of the elevator, and the quadratic integration can obtain the running displacement curve of the elevator.

As can be seen from Figure 5, the downward running speed of the measured elevator is $v_{1}=-1.005 \mathrm{~m} / \mathrm{s}$, the test error is $0.5 \%$, the elevator displacement is $s_{3}=-7.23 \mathrm{~m}$, and the test error is $3.77 \%$. The experimental results show that the instruments and methods used in this experiment can meet the accuracy requirements. This method can be used for emergency brake test of the elevator.

4.5. Test Results of Condition II. Condition II: upward emergency stop condition. This condition is divided into 4 phases, and they are P.1 acceleration phase, P.2 constant speed phase, P.3 emergency braking phase, and P.4 free vibration phase, Figure 7.

Due to the short travel of the tested elevator, in order to ensure the safety of the test, the emergency braking

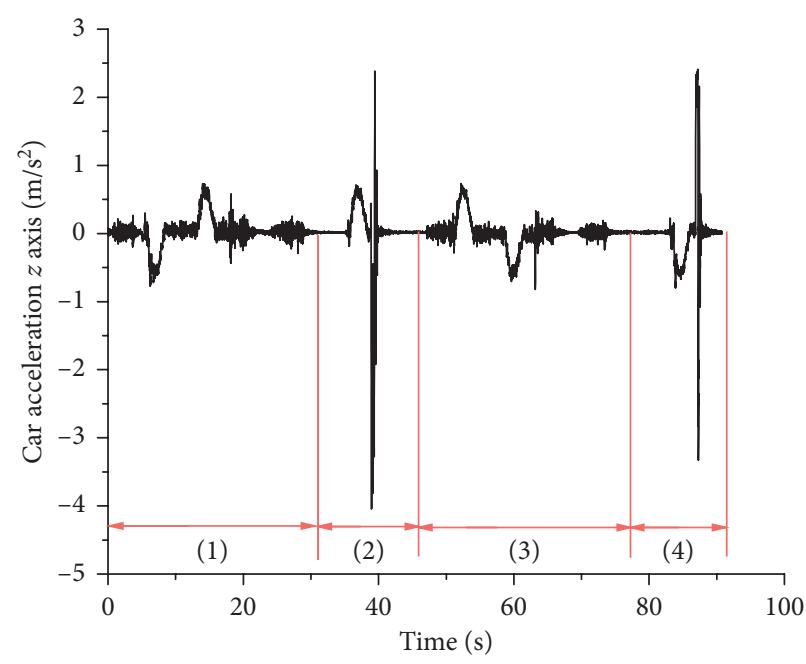

FIgUre 5: Vibration test results. (1) Condition I. (2) Condition II. (3) Condition III. (4) Condition IV.

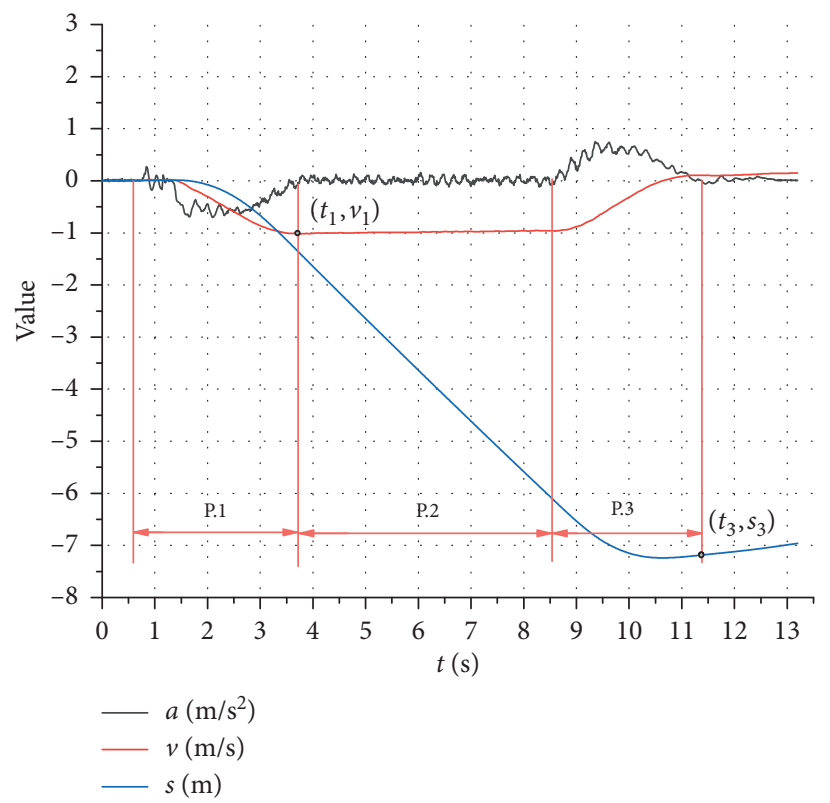

Figure 6: Acceleration, speed, and displacement curve of the elevator car under condition (I) P.1 acceleration phase, P.2 constant speed phase, and P.3 deceleration phase.

operation is carried out immediately after the elevator reaches the rated speed. From Figure 6, it can be seen that, after the normal acceleration of $3.2 \mathrm{~s}$, the speed of the elevator reaches $1.058 \mathrm{~m} / \mathrm{s}$ at the time of $t_{1}=3.58 \mathrm{~s}$. Emergency braking operation is carried out at $t_{2}=3.78 \mathrm{~s}$. The elevator stopped at $t_{3}=4.38 \mathrm{~s}$, the total braking time is $0.6 \mathrm{~s}$, and the braking distance is $s_{3}-s_{2}=0.339 \mathrm{~m}$.

4.6. Test Results of Condition III. Condition III: normal upward working condition. This condition was divided into 3 phases, and they are P.1 acceleration phase, P. 2 constant speed phase, and P.3 deceleration phase, Figure 8 . 


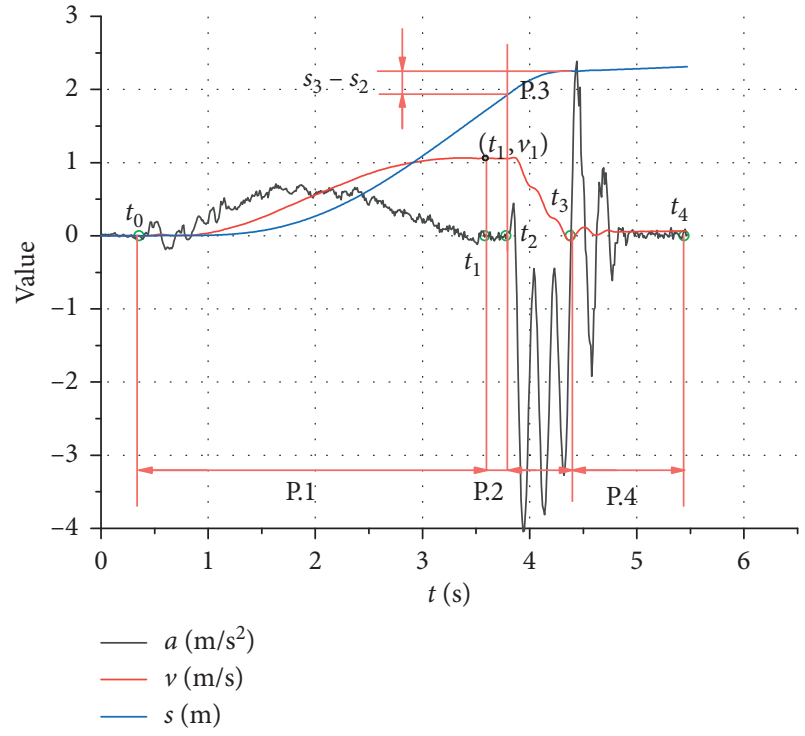

Figure 7: Acceleration, speed, and displacement curve of the elevator car under condition II. P.1 Acceleration phase, P.2 constant speed phase, P.3 emergency braking phase, and P.4 free vibration phase.

As can be seen from Figure 7, the upward running speed of the measured elevator is $v_{1}=1.04 \mathrm{~m} / \mathrm{s}$, the test error is $4.19 \%$, the elevator displacement is $s_{3}=7.77 \mathrm{~m}$, and the test error is $3.47 \%$.

4.7. Test Results of Condition IV. Condition IV: upward emergency stop condition. This condition was divided into 4 phases, and they are P.1 acceleration phase, P. 2 constant speed phase, P.3 emergency braking phase, and P.4 free vibration phase, Figure 9.

Similar to condition II, the emergency braking operation is carried out immediately after the elevator reaches the rated speed. From Figure 8, it can be seen that, after the normal acceleration of $2.8 \mathrm{~s}$, the speed of the elevator reaches $0.98 \mathrm{~m} /$ $\mathrm{s}$ at the time of $t_{1}=3.08 \mathrm{~s}$. Emergency braking operation was carried out at $t_{2}=4.11 \mathrm{~s}$. The elevator stopped at $t_{3}=4.59 \mathrm{~s}$, the total braking time is $0.48 \mathrm{~s}$, and the braking distance is $s_{3}-s_{2}=0.22 \mathrm{~m}$.

\section{Comparison and Analysis}

5.1. Comparison of Test Results of Normal Operation. From the analysis of the test results of normal operation, we can see whether the test method adopted is feasible or not and also know whether the instrument precision adopted can meet the requirements. By comparing the test results of up and down, we can compare the dynamic characteristics of the elevator at different running directions.

As can be seen from Table 3, the test results of working condition III and working condition I are compared, and it is found that the error of the test method adopted is not large in either the ascending or descending stage. When the elevator is descending, the measured running distance is smaller than the actual value; when the elevator is

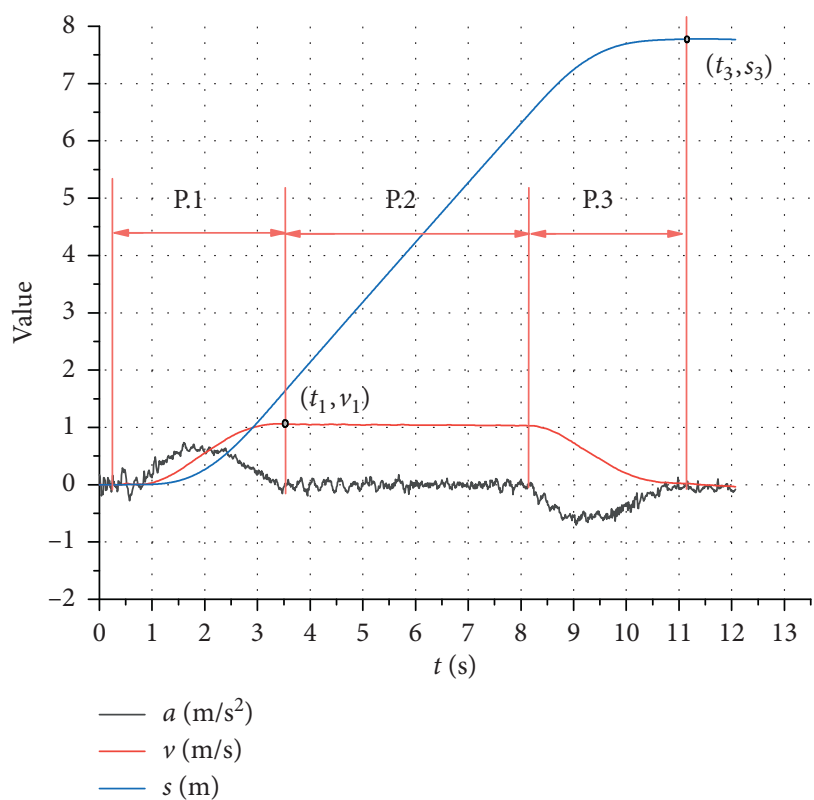

FIGURE 8: Acceleration, speed, and displacement curve of the elevator car under condition III. P.1 Acceleration phase, P.2 constant speed phase, and P.3 deceleration phase.

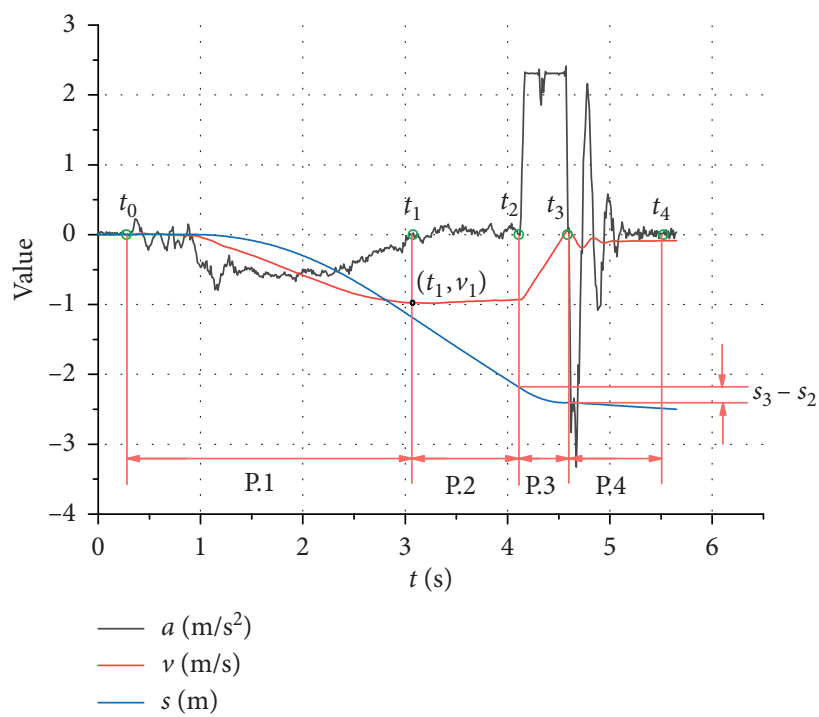

Figure 9: Acceleration, speed, and displacement curve of the elevator car under condition IV. P.1 Acceleration phase, P. 2 constant speed phase, P.3 emergency braking phase, and P.4 free vibration phase.

running upward, the measured running distance is larger than the actual value. This phenomenon may be related to the characteristics of the wire rope, which will be discussed in the comparison of emergency braking test results.

5.2. Comparison of Emergency Brake Test Results. The peak value of the elevator's acceleration during the upward emergency braking process fluctuates greatly, while the 
TABLE 3: Comparison of two normal operation test results.

\begin{tabular}{lcccccc}
\hline $\begin{array}{l}\text { Working } \\
\text { condition }\end{array}$ & \multirow{2}{*}{ Actual speed (m/s) } & Test speed (m/s) & Velocity error (\%) & Actual distance (m) & Test distance (m) & Distance error (\%) \\
\hline Condition I & \multirow{2}{*}{1} & 1.005 & 0.5 & 7.51 & 7.23 & -3.77 \\
Condition III & 1.0419 & 4.19 & \multirow{2}{*}{3.774} & 3.47 \\
\hline
\end{tabular}

downward emergency braking acceleration basically remains at a certain value with no significant change. The upstop distance of the no-load cage is longer than the downstop distance of the no-load cage. This is because, in the upstop process, the heavy gravity is the driving force for the cage, which is not conducive to the deceleration of the elevator. However, in the process of elevator descending and stopping, the weight of gravity becomes the resistance to the car, which is conducive to the deceleration of the elevator. Therefore, during the whole stop process, the car stop time is longer up than down, and the stop distance is also longer.

It can be seen from Figures 6 and 8 that the acceleration variation amplitude of the two working conditions in the emergency braking phase (P.3) is quite different. The main reason for this phenomenon is the different characteristics of the wire rope and the spring. In the emergency braking of the elevator upward (Condition II), the elevator cage will have an upward inertial movement, and the tension of the steel wire rope on the side of the cage will decrease, namely, the steel wire rope will become more relaxed. Therefore, the measured acceleration value changes greatly in the stopping phase (P.3) of Condition II. In the emergency braking of the elevator upward (Condition IV), the elevator cage is affected by the downward inertial force, and the tension of the steel wire rope on the side of the cage increases, namely, the steel wire rope becomes tighter. Therefore, the change of acceleration measured in the stop phase (P.3) of condition IV is almost in a straight line. This also explains why the measured up distance is longer than the measured down distance under normal operating conditions.

Figures 7 and 9 and Table 4 can be summarized as follows.

5.3. Comparison of Theoretical and Experimental Natural Frequencies. For the free vibration phase (P.4) of working conditions II and IV, the brake of the elevator has been stable, and the vibration of the elevator traction system can be considered as free vibration. The amplitude-frequency curve of elevator free vibration measured in the experiment is shown in Figure 10.

According to the comparison between the natural frequency theoretically calculated by the three-degree-offreedom system model and the natural frequency measured by the experiment, it can be seen that the measured value of the first natural frequency (MF1) is 0 because of the rigid body displacement of the system, which is consistent with the theoretical value of the first natural frequency (TF1). The measured value of the second natural frequency (MF2) is about $5.4 \mathrm{~Hz}$, and the measured value of the third natural frequency (MF3) is 34. Theoretical value and measured value of natural frequency are shown in Table 5.
TABLE 4: P.3 comparison of parameters measured during emergency braking.

\begin{tabular}{lcccc}
\hline $\begin{array}{l}\text { Working } \\
\text { condition }\end{array}$ & $\begin{array}{c}\text { Time } \\
\text { duration } \\
(\mathrm{s})\end{array}$ & $\begin{array}{c}\text { Peak } \\
\text { acceleration } \\
\left(\mathrm{m} / \mathrm{s}^{2}\right)\end{array}$ & $\begin{array}{c}\text { Initial } \\
\text { velocity } \\
(\mathrm{m} / \mathrm{s})\end{array}$ & $\begin{array}{c}\text { Stopping } \\
\text { distance } \\
(\mathrm{m})\end{array}$ \\
\hline $\begin{array}{l}\text { Condition } \\
\text { II }\end{array}$ & 0.60 & 4.00 & 1.06 & 0.339 \\
$\begin{array}{l}\text { Condition } \\
\text { IV }\end{array}$ & 0.48 & 2.41 & 0.98 & 0.222 \\
\hline
\end{tabular}

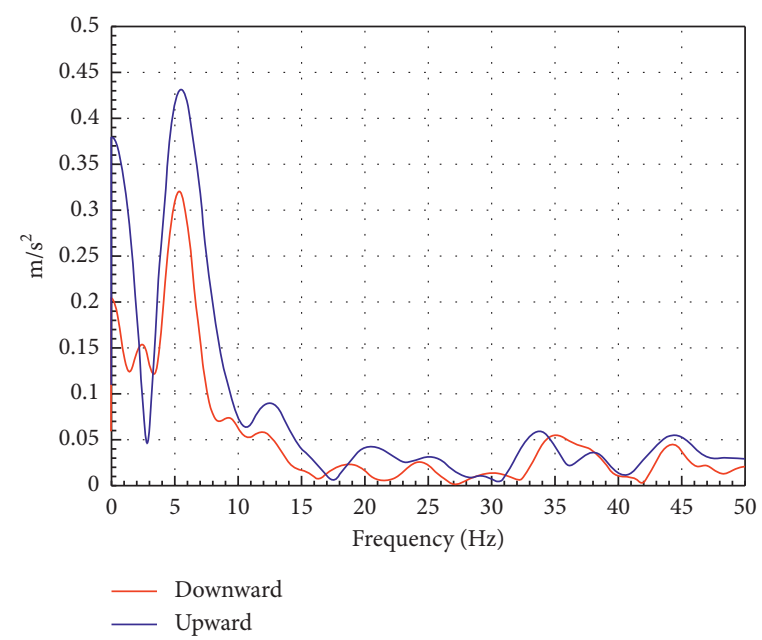

FIGURe 10: Spectrum analysis of P.4 under conditions II and IV.

TABLE 5: Theoretical value and measured value of natural frequency.

\begin{tabular}{lcc}
\hline Experimental running direction & Upward & Downward \\
\hline Cage position (relative to the top floor) $(\mathrm{m})$ & 5.26 & 2.42 \\
TF1 & 0 & 0 \\
MF1 & 0 & 0 \\
TF2 & 7.05 & 7.01 \\
MF2 $(\mathrm{Hz})$ & 5.49 & 5.38 \\
MF2/TF2 *100\% (\%) & 77.87 & 76.74 \\
TF3 & 25.61 & 32.20 \\
MF3 & 33.82 & 35.05 \\
MF2/TF2 $* 100 \%(\%)$ & 132.06 & 108.85 \\
\hline
\end{tabular}

The analysis results show that the three DOF model can basically meet the accuracy requirements for the dynamic analysis of the elevator with low lifting height, and the theoretical solution can reflect the engineering practice. 


\section{Conclusion}

By analyzing the vibration of the elevator traction system, this paper establishes the vibration characteristic calculation model of the multi-DOF system. After a reasonable simplification of the model, a 3-DOF system is used to analyze the vibration response characteristics of the elevator traction system under emergency braking. This paper also puts forward a test method for the characteristics of elevator emergency braking vibration and tests and analyzes the operation of an elevator with a lifting height of 7.51 meters in the laboratory under four different working conditions. Based on these works, the following conclusions are obtained:

(1) From the comparison of the normal uplink and downlink test results, it can be seen that the speed and distance errors measured by the test method proposed in this paper are not more than $4.2 \%$, no matter in the uplink or downlink stage, which shows that the test method adopted in this paper is correct and the instrument used has high precision. It is also found that the measured running distance is smaller than the actual value when the elevator moves downward and larger than the actual value when the elevator moves upward.

(2) Due to the force characteristics of the steel wire rope, the operation characteristics of the elevator cage are not the same in the upward and downward conditions. The vibration in the no-load ascending emergency braking process is significantly greater than that in the descending process, and the ascending emergency braking peak is 1.66 times of the descending peak. The upward braking distance is 1.53 times that of the downward braking distance, so we should pay more attention to the upstream emergency braking characteristics when analyzing the safety situation of the no-load operation of the elevator.

(3) For elevators with small lifting height, the 3-DOF centralized mass discretization model is adopted for analysis without the compensation rope. The model adopted can accurately calculate the natural frequency of the system, and the difference between the natural frequency calculated in theory and the measurement is about $30 \%$. It shows that the simplified method can meet the requirement of accuracy.

(4) By comparison with experiments, it can be seen that the analysis model of elevator vibration characteristics proposed in this paper can reflect the impact of emergency braking excitation provided by elevator brakes on the vibration of the elevator cage, counterweight, and traction wheel. It can be used for the analysis of elevator braking performance and has great reference value for the safety performance research of the elevator traction system.

\section{Data Availability}

The data used to support the findings of this study are available from the corresponding author upon request.

\section{Conflicts of Interest}

The authors declare that they have no conflicts of interest.

\section{Acknowledgments}

The authors gratefully acknowledge the financial support provided by the Science and Technology Scheme of Guangzhou City (no. 201904010141) and the Guangzhou Market Supervision Administration Bureau (no. 2019KJ06).

\section{References}

[1] S. Cao, R. Zhang, S. Zhang, S. Qiao, D. Cong, and M. Dong, "Roller-rail parameters on the transverse vibration characteristics of super-high-speed elevators," Transactions of the Canadian Society for Mechanical Engineering, vol. 43, no. 4, pp. 535-543, 2019.

[2] G. Wang, X. Xiao, and Y. Liu, "Dynamic modeling and analysis of a mine hoisting system with constant length and variable length," Mathematical Problems in Engineering, vol. 2019, Article ID 4185362, 12 pages, 2019.

[3] Q. Zhang, Z. Yang, C. Wang, Y. Yang, and R. Zhang, "Intelligent control of active shock absorber for high-speed elevator car," Proceedings of the Institution of Mechanical Engineers, Part C: Journal of Mechanical Engineering Science, vol. 233, no. 11, pp. 3804-3815, 2019.

[4] J. Liu, R. Zhang, Q. He, and Q. Zhang, "Study on horizontal vibration characteristics of high-speed elevator with airflow pressure disturbance and guiding system excitation," $M e$ chanics and Industry, vol. 20, no. 3, 2019.

[5] P. Wolszczak, P. Lonkwic, A. Cunha Jr., G. Litak, and S. Molski, "Robust optimization and uncertainty quantification in the nonlinear mechanics of an elevator brake system," Meccanica, vol. 54, no. 7, pp. 1057-1069, 2019.

[6] T. X. Nguyen, N. Miura, and A. Sone, "Analysis and control of vibration of ropes in a high-rise elevator under earthquake excitation," Earthquake Engineering and Engineering Vibration, vol. 18, no. 2, pp. 447-460, 2019.

[7] S. Watanabe and T. Okawa, "Vertical vibration of elevator compensating sheave due to brake activation of traction machine," Journal of Physics: Conference Series, vol. 1048, no. 1, pp. 1-7, 2018.

[8] R. Roberts, "Control of high-rise/high-speed elevators," in Proceedings of the 1998 American Control Conference, IEEE, Philadelphia, PA, USA, June 1998.

[9] Z. Yang, Q. Zhang, R. Zhang, and L. Zhang, "Transverse vibration response of a super high-speed elevator under air disturbance," International Journal of Structural Stability and Dynamics, vol. 19, no. 9, 2019.

[10] N. V. Gaiko and W. T. van Horssen, "Resonances and vibrations in an elevator cable system due to boundary sway," Journal of Sound and Vibration, vol. 424, pp. 272-292, 2018.

[11] W. Fan and W. D. Zhu, "Dynamic analysis of an elevator traveling cable using a singularity-free beam formulation," Journal of Applied Mechanics-Transactions of the ASME, vol. 84, no. 4, 2017.

[12] E. Durak and H. A. Yurtseven, "Experimental study of the tribological properties of an elevator's brake linings," Industrial Lubrication and Tribology, vol. 68, no. 6, pp. 683-688, 2016.

[13] L. Xiao, "Failure mechanism analysis of elevator brake," in Proceedings of the 2017 4th International Conference on Machinery, Materials and Computer (MACMC 2017), vol. 150, pp. 405-409, Xi'an, China, November 2017. 
[14] P. Lonkwic, "Influence of friction drive lift gears construction on the length of braking distance," Chinese Journal of $\mathrm{Me}$ chanical Engineering, vol. 28, no. 2, pp. 363-368, 2015, in Chinese.

[15] Q. Peng, Z. Li, H. Yuan, G. Huang, S. Li, and X. Sun, "A model-based unloaded test method for analysis of braking capacity of elevator brake," Advances in Materials Science and Engineering, vol. 2018, Article ID 8047490, 10 pages, 2018.

[16] R. Longwic and K. Szydło, "The impact of the elevator guides contamination on the braking process delay for selected progressive gears," Advances in Science and Technology Research Journal, vol. 11, no. 2, pp. 1-7, 2017.

[17] C. Li, H. Chunli, J. Qin, and Z. Zhu, "Research on the dynamic characteristics of high-speed elevator system," in Proceedings of the 2019 International Confernce on Electronical, Mechanical and Materials Engineering (ICE2ME 2019), vol. 181, pp. 105-109, Wuhan, China, January 2019.

[18] R. Zhang, C. Wang, Q. Zhang, and J. Liu, "Response analysis of non-linear compound random vibration of a high-speed elevator," Journal of Mechanical Science and Technology, vol. 33, no. 1, pp. 51-63, 2019.

[19] Ji Wang, S. Koga, Pi Yangjun, and K. Miroslav, "Axial vibration suppression in a partial differential equation model of ascending mining cable elevator," Journal of Dynamic Systems Measurement and Control-Transactions of the ASME, vol. 140, no. 11, 2018.

[20] J.-H. Shin, E. Talib, and M. K. Kwak, "Vibration of a string against multiple spring-mass-damper stoppers," Journal of Sound and Vibration, vol. 414, pp. 157-173, 2018.

[21] N. Wang, G. Cao, Y. Lu, and L. Wang, "Modeling and control for a multi-rope parallel suspension lifting system under spatial distributed tensions and multiple constraints," Symmetry-Basel, vol. 10, no. 9, 2018.

[22] X. Jiang, L. Guo, S. Niu, and Y. Rui, "Vibration analysis and simulation of traction inclined elevator," in Proceedings of the 6 th International Workshop of Advanced Manufacturing and Automation, vol. 24, pp. 221-224, Manchester, UK, November 2016.

[23] R. S. Crespo, S. Kaczmarczyk, P. Picton, and H. Su, "Modelling and simulation of a stationary high-rise elevator system to predict the dynamic interactions between its components," International Journal of Mechanical Sciences, vol. 137, pp. 24-45, 2018.

[24] K. Batraz, "Sustainable limitation of high-frequency oscillations of elevator cabin," in Proceedings of the Energy Management of Municipal Transportation Facilities and Transport (EMMFT 2017), vol. 90, Khabarovsk, Russia, April 2017.

[25] R.-jun Zhang, C. Wang, and Q. Zhang, "Response analysis of the composite random vibration of a high-speed elevator considering the nonlinearity of guide shoe," Journal of the Brazilian Society of Mechanical Sciences and Engineering, vol. 40, no. 4, 2018. 